\title{
Un enfoque diferente para la resolución de problemas
}

\section{Elena Pérez-Bernabeu ${ }^{\mathrm{a}}$, M.A. Sellés ${ }^{\mathrm{b}}$, V.Gisbert $^{\mathrm{c}}$, S. Sánchez-Caballero ${ }^{\mathrm{d}}$}

${ }^{a}$ Departamento de Estadística e Investigación Operativa Aplicadas y Calidad. Campus de Alcoy. Universidad Politécnica de Valencia. E-mail: elenapb@eio.upv.es, ${ }^{\mathrm{b}}$ Departamento de Ingeniería Mecánica y de Materiales. Campus de Alcoy. Universidad Politécnica de Valencia. E-mail: maselles@dimm.upv.es, ${ }^{c}$ Departamento de Estadística e Investigación Operativa Aplicadas y Calidad. Campus de Alcoy. Universidad Politécnica de Valencia. E-mail: vgisber@eio.upv.es, y ${ }^{\mathrm{d}}$ Departamento de Ingeniería Mecánica y de Materiales. Campus de Alcoy. Universidad Politécnica de Valencia. Email: sasanca@dimm.upv.es.

\begin{abstract}
When solving problems skills are involved in some specific courses, it turns out fundamental that students develop the ability of understanding the assignment texts. We have realized that students, in general, have a lack in the comprehension of texts, particularly of problem texts. In our case, we have made a proposal using a methodology for students who attend statistics topic courses in engineering degrees, so that it makes easier for them to do a critical reading of the assignments, but bearing in mind that the aim of the course is not that they learn "to read" but statistics. It was offered as a voluntary activity including the incentive that they would be able to gain an additional mark so that it could be posible to increase the final grade obtained by usual ways. The students response was considered positive, so the experience is going to be replicated in following academic years. In addition, we implemented other innovative methodologies so as to promote generic competencies as oral and written communication skills in the own language, as well as in another foreign language (in this case, the English language).
\end{abstract}

Keywords: Problem solving, Active Participation, Reading Comprehension.

\footnotetext{
Resumen

En las asignaturas en las que está involucrada la capacidad de resolución de problemas resulta fundamental que los alumnos desarrollen la habilidad de entender los enunciados de los problemas. Se ha detectado que el alumnado en general tiene carencias en la comprensión de textos, particularmente de enunciados de problemas. En nuestro caso concreto, se ha propuesto una metodología para los alumnos que cursan asignaturas de estadística en grados de ingeniería, de modo que les facilite una lectura crítica de estos enunciados, pero teniendo en cuenta que el objetivo de las asignaturas no es que aprendan a 'leer', sino estadística. Se ofertó como una actividad voluntaria con el incentivo de poder ganar una nota adicional que les permitiese aumentar la calificación obtenida mediante las vías tradicionales. La respuesta por parte del alumnado se consideró positiva, por lo que se va a repetir el esfuerzo en los próximos cursos. Además, se integró con otras metodologías innovadoras
} 
que fomentaban competencias genéricas tales como la comunicación oral y escrita en la propia lengua, así como en otra lengua extranjera (en este caso, el inglés).

Palabras clave: Resolución de problemas, Participación Activa, Comprensión Lectora.

\section{Introducción}

La resolución de problemas se ha considerado clave en la enseñanza de ciencias experimentales, de modo que no se podría entender su estudio sin poder probar que la teoría funciona. La didáctica de las ciencias experimentales conlleva implícitamente la habilidad de resolución de problemas.

Cunnington, B (1985) ya afirmaba que existía un conflicto entre la cultura académica y la cultura empresarial, por lo que en el entorno académico prima la teoría y se da mayor importancia al conocimiento y la comprensión, en cambio, en la cultura empresarial resulta primordial poder demostrar destrezas y se centra principalmente en la práctica.

Es por lo mencionado anteriormente que la resolución de problemas resulta clave para hacer de puente entre la cultura académica y la cultura empresarial, ya que aúna en una misma actividad las habilidades requeridas en ambos mundos.

En nuestro caso, tratando materias como la estadística, los problemas y su resolución forman parte intrínseca de la materia en si. Polya, G. (2014) detalla las fases de la resolución de problemas como eje central de los métodos matemáticos, que sostuvo ya en la primera edición de este libro, en 1945, y que se pueden ver en la Tabla 1.

Tabla 1. Etapas de la resolución de problemas.

\section{ETAPAS DE LA RESOLUCIÓN DE PROBLEMAS}

1. Entender y explorar el problema

2. Encontrar una estrategia.

3. Utilizar la estrategia para resolver el problema.

4. Volver atrás y reflexionar sobre la solución.

Fuente: Polya, G (2014)

La primera de las etapas que sugiere Polya, G. (2014) es 'entender y explorar el problema', y es en esta fase en la que hay que incidir mucho, puesto que es el pilar en el que se asientan las demás etapas. Sin una adecuada comprensión del problema, no podrá localizarse ninguna estrategia, ni resolverlo utilizando la misma.

\section{Objetivos}

En este nuevo enfoque para la resolución de problemas se trata de que los alumnos pierdan el temor a la resolución de problemas complejos mediante un cambio de roles. El alumno pasa de resolver problemas a plantear los que ha de resolver tras haber resuelto ejercicios previamente propuestos por el docente. Este planteamiento surgió a raíz

2016, Universitat Politècnica de València 
de los problemas que los estudiantes tienden a sufrir en la comprensión de los enunciados, que implica en ocasiones una riqueza léxica reducida así como también falta de base a nivel gramatical y ortográfico. Estas carencias de nivel transversal no se pueden suplir durante un cuatrimestre de docencia de una asignatura que no es de letras, sino más bien números, pero sí se pueden fijar las estructuras más habituales de composición de enunciados y a la vez, aprender a identificar la información necesaria para la resolución de los ejercicios.

No se puede presuponer que los enunciados van a hacer uso de un lenguaje sencillo y claro, como indica Perales, F.J. (2000). Es por eso que uno de los objetivos también es proveer al alumno de herramientas lingüística, tales como un vocabulario específico de la materia, así como más generales, que incluyen la comprensión detallada de estructuras gramaticales comúnmente empleadas

\section{Desarrollo de la innovación}

Este planteamiento se ha puesto en marcha en la asignatura 'Estadística' del grado de Ingeniería Informática durante el segundo semestre del curso 2013-14. Se propuso a los alumnos que redactasen enunciados sobre el tema que acaban de ver en clase, basándose en los ejercicios que se habían resuelto durante las mismas, pero sin ser copias exactas de estos, ni obtenerse directamente de internet.

Como esta actividad se consideró voluntaria, no creó presión a los alumnos que participaron, que pudieron crear tantos ejercicios nuevos como consideraron. Como el objetivo fue que comprendiesen cómo se plantean ejercicios y, a la vez, cuáles son los datos más importantes para cada tipo de ejercicios. También supone una mayor implicación del alumno con una participación más activa en la asignatura. Se ha observado que los estudiantes que participaron en esta actividad voluntaria han sido capaces de comprender mejor los enunciados tras una primera lectura, y se han centrado mucho más en extraer la información relevante del mismo que permitiese una resolución correcta. Además, estos alumnos han comprendido mejor los conceptos implicados en el tema tras haber planteado estos ejercicios.

El Aprendizaje Basado en Problemas se ha postulado como una de las metodologías en auge, es por lo que se promociona también en esta asignatura, logrando además una mayor participación del alumnado en la impartición de la materia.

Aprender a pensar es una actividad que no debemos pasar por alto si pretendemos que los alumnos adquieran una capacidad elevada de resolución de problemas, y está íntimamente ligada a la comprensión lectora, puesto que el hecho de pensar tiene que estar vinculado a tener claro qué problema han de resolver.

Por otra parte, también se considera necesario un aprendizaje activo por parte del alumno. Mediante el desarrollo de esta metodología se consigue implicar más al alumno en la impartición de la asignatura, ya que se siente parte del proyecto global de la misma.

Finalmente, los ejercicios de nueva creación que proponían los alumnos se distribuyeron a través de la plataforma en línea de la que dispone la Universitat Politècnica de València, POLIFORMAT. Se hizo de forma anónima, para que los alumnos que habían participado no se sintiesen expuestos frente al resto de alumnos que no habían participado. Así, con la información de estos enunciados se hizo un recopilatorio para que todos los alumnos de clase tratasen de resolver los problemas planteados desde la mente de un estudiante como ellos. Además, como incentivo adicional para todo el grupo, se indicó que, al menos uno de los

(cc) EY-NC-ND 2016, Universitat Politècnica de València

Congreso In-Red (2016) 
problemas propuestos por los compañeros saldría en el examen de esa parte de la asignatura. En general se detectó un aumento de las tutorías atendidas en el momento se publicaron los ejercicios propuestos por los propios estudiantes. Esto se explica porque las soluciones no están disponibles de otros cursos anteriores, y con el aliciente de que alguno de esos ejercicos iba a estar propuesto como ejercicio de examen, trataron de resolver estos ejercicios con mayor entusiasmo.

Tabla 2. Datos sobre el grupo piloto.

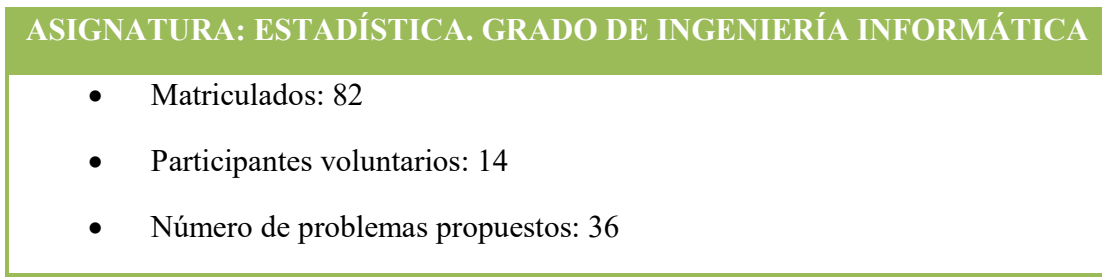

Fuente: Elaboración propia

Como resumen, vemos en la tabla 2 información sobre los alumnos que participaron respecto a los matriculados.

\section{Resultados}

Los resultados obtenidos en esta primera fase fueron prometedores, en cuanto se consiguió una mejora de los resultados de aprendizaje, así como motivación, participación y satisfacción del profesorado y de los alumnos. Se percibió como un reto, que tanto los alumnos como el profesorado fomentó en las distintas sesiones de clase.

Se muestra en la tabla 3 un resumen de las calificaciones obtenidas por el grupo piloto a modo de indicador de funcionamiento de la innovación docente.

Tabla 3. Calificaciones obtenidas por el grupo piloto.

\begin{tabular}{|c|c|c|}
\hline Calificación & $\mathbf{N}^{0}$ Alumnos & Porcentaje \\
\hline Suspensos & 16 & $19,5 \%$ \\
\hline No presentados & 6 & $7,3 \%$ \\
\hline $5 \leq$ Nota $<6$ & 21 & $25,6 \%$ \\
\hline $6 \leq$ Nota $<7$ & 16 & $19,5 \%$ \\
\hline $7 \leq$ Nota $<8$ & 15 & $18,3 \%$ \\
\hline $8 \leq$ Nota $<9$ & 5 & $6,1 \%$ \\
\hline Nota $\geq 9$ & 3 & $3,7 \%$ \\
\hline
\end{tabular}

Fuente: Elaboración propia

(cc) EY-NC-ND 2016, Universitat Politècnica de València

Congreso IN-RED (2016) 
En la tabla 3 se observa que aprobaron la asignatura un 73,2\% de los alumnos inicialmente matriculados, teniendo en cuenta que siempre hay algunos alumnos que no se presentan a la asignatura por diversos motivo, que en este caso es de un 7,3\%. Si no consideramos en los cálculos a los alumnos que no se presentaron a la asignatura, entonces cerca del $80 \%$ aprobaron la misma. Se ha de tener en cuenta también que es una asignatura que tradicionalmente cuesta de aprobar, por tener un alto índice de alumnos provenientes de módulos de formación profesional en este grado que suelen tener una formación menos intensa en este tipo de materias básicas. Además, de los 14 alumnos voluntarios que participaron, 12 de ellos obtuvieron una calificación superior a 7, incluyendo 2 matrículas de honor.

Aunque el porcentaje de participación en la propuesta de ejercicios no se puede considerar elevada -queda por debajo del $20 \%$ de los alumnos matriculados en la asignatura-, realmente muchos más alumnos participaros en la segunda etapa de la propuesta de ejercicios, ya en la resolución de los mismos. No existen datos fiables sobre quién resolvió estos ejercicios, pero se estima que el interés fue muy alto por la expectación creada, y por el incremento en la solicitud de citas para tutorías al respecto.

Por otra parte, durante el mes de Mayo de 2016 se pasó una encuesta a los alumnos que participaron, obteniéndose los resultados mostrados en la figura 1:

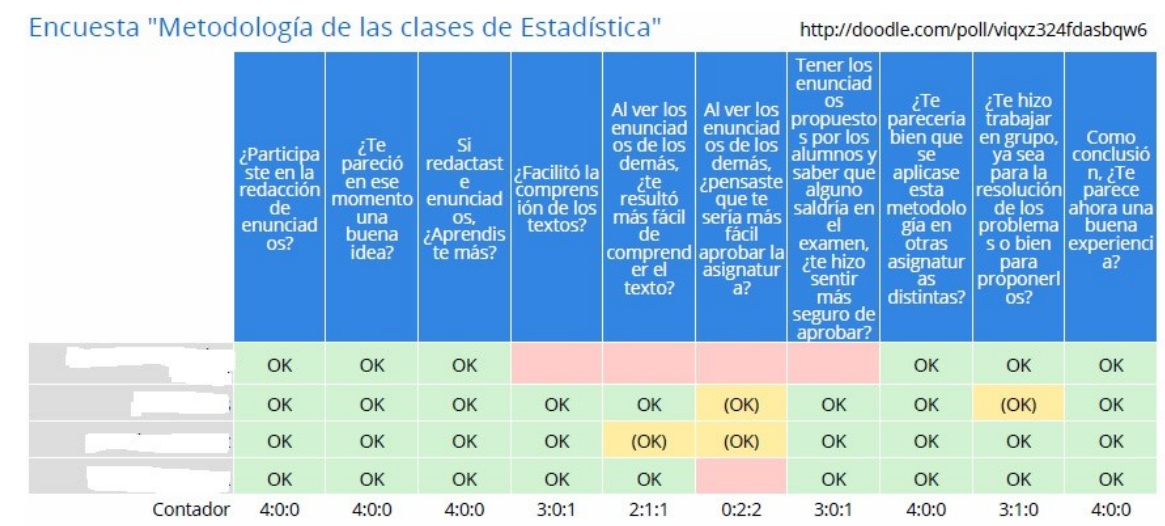

Figura 1. Encuesta sobre la percepción de la innovación dos años después.

Para encuestar a los alumnos se envió un enlace a una encuesta generada con ww.doodle.com a los alumnos que participaron, dos años después de haberse puesto en marcha, obteniéndose los resultados mostrados en la figura 1. La participación en esta encuesta no se puede considerar significativa, puesto que solamente han participado 4 alumnos del grupo que además participaron en la propuesta de ejercicios. Aún así se ha considerado conveniente mostrar la percepción de esta muestra de alumnos que aceptaron participar en la encuesta, teniendo en cuenta que la totalidad obtuvieron notas entre notable y sobresaliente M.H., Todos ellos coinciden en que sería bueno que se aplicase a otras asignaturas, ya que les pareció una buena experiencia -tanto en ese momento, hace dos años, como en la actualidadasí como consideran que aprendieron más cuando redactaron nuevas propuestas de ejercicios. Aunque esto no es óbice para que aún así no se considerase una gran ventaja para aprobar la asignatura.

(cc) EY-NC-ND 2016, Universitat Politècnica de València

Congreso In-Red (2016) 
Se desarrollan competencias genéricas de varios tipos:

1.- Instrumentales, puesto que en la redacción de las propuestas intervienen todos los aspectos relacionados con la comunicación escrita, así como discurren cómo se pueden resolver aplicando los conocimientos de los que disponen :

- Comunicación oral y escrita en la propia lengua.

- Capacidad de análisis y síntesis.

- Resolución de problemas.

- Conocimientos generales básicos.

2.- Interpersonales :

- Capacidad crítica y autocrítica. Durante la redacción de los ejercicios tuvieron que 'autoregularse' para conseguir ejercicios resolubles y ellos mismos pudiesen comprenderlos.

3.- Sistémicas :

- Capacidad de aplicar los conocimientos en la práctica.

- Motivación de logro, en cuanto se sintieron realizados, tanto al realizar la actividad como al ver que sus compañeros resolvieron los problemas por ellos planteados.

- Habilidad para trabajar de forma autónoma.

- Capacidad de aprender.

El profesorado percibió una mejora en la eficacia a la hora de asimilar conceptos, así como una participación muy activa de los alumnos voluntarios que se prestaron a realizar esta actividad, hecho que les facilitó el auto-aprendizaje, así como todos los alumnos que intentaron resolver los mismo de forma individual o en grupo.

\section{Conclusiones}

Como conclusiones, podemos afirmar que se trata de una metodología poderosa para tratar de que los alumnos obtengan una mejor resiliencia de los conocimientos transmitidos a lo largo de la asignatura. Pero no por ello puede recaer en ella la responsabilidad de enseñar a los alumnos a pensar e interpretar los enunciados, ha de combinarse con otras técnicas que permitan asegurar un correcto entendimiento de los textos, así como un análisis crítico de la información transmitida.

Por otra parte, en la universidad en general se está haciendo hincapié en el desarrollo de competencias transversales, que habrían de reforzarse como las competencias genéricas que se mencionan en el punto anterior, puesto que se detectan carencias en la comprensión de textos básicos a nivel universitario y también en el nivel de comunicación en la propia lengua.

\section{Referencias}

CUNNINGTON, B. (1985). "Management development and education process" en Journal of Management Development. Vol. 4, núm. 5.

PERALES PALACIOS, F.J. (2000). "La resolución de problemas" en Perales Palacios, F.J. y añal de León, P. Didáctica de de las ciencias experimentales. Alcoy: Editorial Marfil.

POLYA, G. (2014). How to Solve It: A New Aspect of Mathematical Method. Princeton University Press. 